\title{
Occlusive Therapy in Atopic Dermatitis
}

\author{
Misha M. Heller ${ }^{1}$, Eric S. Lee ${ }^{2}$, Faranak Kamangar ${ }^{3}$, \\ Wilson Liao ${ }^{4}$ and John Y. M. Koo ${ }^{4}$ \\ ${ }^{1}$ University of Southern California, Keck School of Medicine, Los Angeles, California \\ ${ }^{2}$ University of Nebraska Medical Center, College of Medicine, Omaha, Nebraska \\ ${ }^{3}$ University of California Davis, School of Medicine, Sacramento, California \\ ${ }^{4}$ University of California San Francisco, Department of Dermatology \\ San Francisco, California \\ USA
}

\section{Introduction}

The treatment of atopic dermatitis (AD) can be challenging for dermatologists and other healthcare professionals. Conventional treatments consisting of emollients and topical corticosteroids are often insufficient for severe and/or refractory AD. Other therapies include systemic corticosteroids, photochemotherapy using psoralen and ultraviolet-A light, and cyclosporine. However, all of these approaches have potentially serious side effects and relative contraindications, especially in children. Over the past two decades, occlusive therapy has been advocated as a safe and effective treatment modality for individuals with acute erythrodermic $\mathrm{AD}$ and those with severe and/or refractory AD. (Nicol 1987; Goodyear et al. 1991; Bridgman 1995; Devillers and Oranje 2005)

Occlusive therapy usually involves the application of emollients, antiseptics, or topical corticosteroids under either wet wrap dressings (WWD) or dry wrap dressings (DWD). This consists of moistened open-weave cotton tubular bandages (eg. Tubifast ${ }^{\circledR}$, Tubigauz ${ }^{\circledR}$ ) in WWD occlusion, versus dry gauze, plastic wraps, or hydrocolloid dressings (eg. Duoderm ${ }^{\circledR}$ ) in DWD occlusion. (Nicol 1987; Goodyear et al. 1991; Bridgman 1995)

More recently, however, occlusion alone using a hydrogel patch has been utilized. The theory is that a major component in the pathophysiology of $\mathrm{AD}$ is barrier dysfunction. In fact, many current therapies target this barrier defect (i.e., pseudoceramide moisturizers and skin barrier emulsions). As such, an ideal repair mechanism would completely eliminate microbe and allergen penetration and transepidermal water loss in AD, both of which lead to xerosis, hypersensitivity, pruritus, and inflammation. The hydrogel patch, therefore, offers an innovative approach to complete barrier repair. (Park et al. 2011)

Regardless of the treatment approach, occlusive therapy offers many advantageous such as a cooling effect on inflamed skin, increased penetration of topical agents, enhanced skin hydration, and a barrier to external antigens and trauma (i.e. scratching). Reported disadvantages include the cumbersome and time-consuming nature of the application process, risk of allergic reaction to the occlusive material itself, and possible increased risk of infectious complications such as folliculitis, furunculosis, or cellulitis. An additional concern is that occlusion may cause skin maceration if used incorrectly, or can paradoxically 
promote skin dryness if too little topicals are applied. (Nicol 1987; Goodyear et al. 1991; Bridgman 1995; Krakowski et al. 2008)

Occlusion alone of both normal and lesional eczematous skin can result in increased density of cutaneous microbial flora. (Aly et al. 1978; Rajka et al. 1981) Moreover, there is a positive association between Staphylococcus aureus (S. aureus) colonization and disease severity. (Williams 2000) These findings suggest that occlusion, by increasing the density of S. aureus, might push already colonized eczematous skin into the realm of clinical infection. In addition, innate production of cutaneous anti-microbial peptides, such as sphingosine and $\beta$-defensin, is depressed in patients with AD such that eczematous skin possesses decreased natural resistance to bacterial invasion (Arikawa et al. 2002; Ong et al. 2002) Thus, evidence of clinically apparent skin infection may be a contraindication to occlusive therapy, as it may exacerbate the infection. (Aly et al. 1978) However, the possibility that $S$. aureus colonization may complicate occlusive therapy has not been adequately addressed to date.

Another concern is that the use of topical corticosteroids under occlusion may relate to an increased potential for absorption and greater incidences of possible adverse effects. Most attention has been focused on the risk of skin atrophy and striae, hypothalamic-pituitaryadrenal (HPA) axis suppression, as well as growth impairment in children.

Ultimately, this chapter aims to examine current evidence on the safety and efficacy of occlusive therapy in the treatment of AD.

\section{Methods}

Studies on the use of occlusive therapy for the treatment of atopic dermatitis were identified in PubMed and Embase Medline databases from January 1966 to April 2011, using the key terms "occlusion," "occlusive dressings", "occlusive therapy," "wet wrap," "wet dressings," "dry wrap," "dry dressings," "atopic dermatitis," "dermatitis," and "eczema." Key terms were also searched in combination. Reference lists of relevant publications were manually searched for additional relevant studies. The search was limited to original studies and review articles published in English or with English abstracts, in humans. Studies of small size and those that did not use a controlled or randomized study design were included due to the dearth of published literature on this topic. Publications meeting these criteria were then reviewed for study design, population, disease severity or type, study size, efficacy, and safety. The topical agent used, dilution, and type and period of occlusive therapy were also noted, when such information was made available in the publication. In addition, response to therapy was seen as improvement in disease severity or symptoms from baseline, improvement in SCORing Atopic Dermatitis (SCORAD), and/or improvement in Eczema Area and Severity Index (EASI) score, depending on what information was provided. Of note, SCORAD is composite score of eczema severity based on the surface area involved, intensity of symptoms, and subjective symptoms such as sleep disturbance and pruritus. EASI is a 12-point scoring system of disease severity assessing erythema, induration, lichenification, pruritus, and excoriation. Finally, conclusions were drawn from the results of these studies bearing in mind standard clinical practice.

\section{Studies on occlusive therapy}

There are a total of 19 studies evaluating the use of occlusive therapy in AD (Table 1). Fourteen studies used WWD, 5 of which were randomized controlled trials. All WWD 


\begin{tabular}{|c|c|c|c|c|c|c|c|c|c|c|}
\hline \multirow[t]{2}{*}{ |a } & 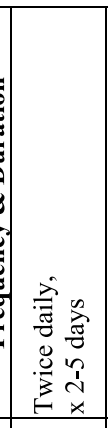 & 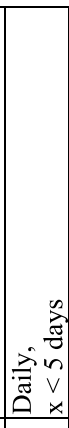 & 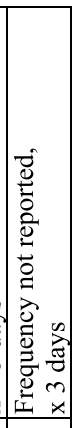 & 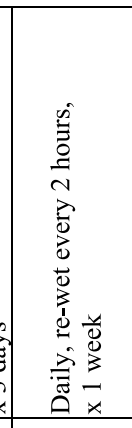 & 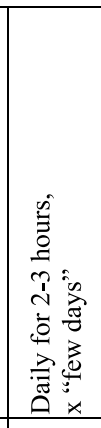 & 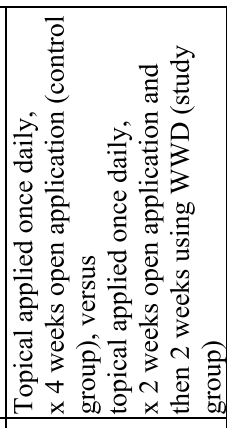 & 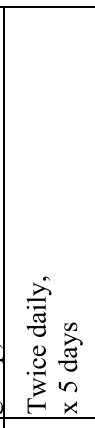 & 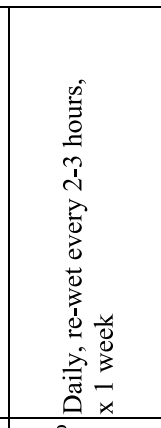 & 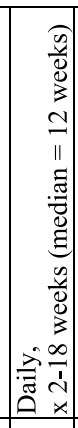 & 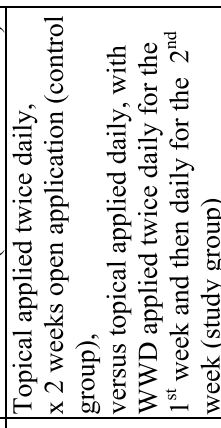 \\
\hline & 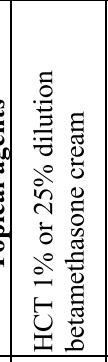 & 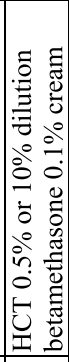 & 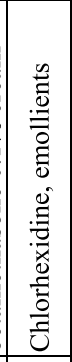 & 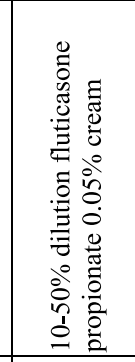 & 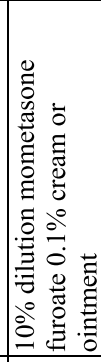 & 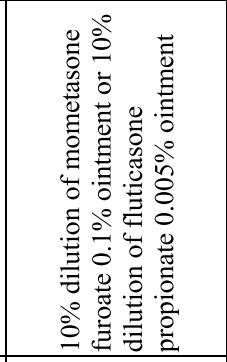 & 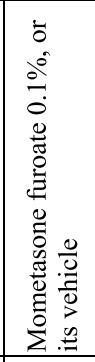 & 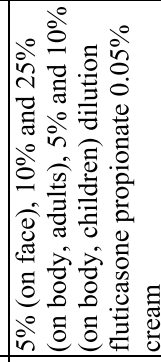 & 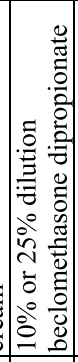 & $\stackrel{\circ}{\circ}$ \\
\hline 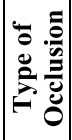 & $\sum_{3}^{\infty}$ & $\sum_{i}^{2}$ & $\sum_{3}^{2}$ & $\sum_{3}^{\theta}$ & $\sum_{i}^{2}$ & $\sum_{3}^{\theta}$ & $\sum_{3}^{2}$ & 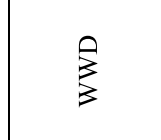 & $\sum_{i}^{2}$ & $\sum_{3}^{2}$ \\
\hline 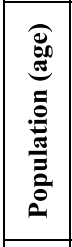 & 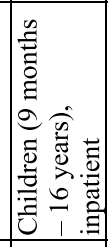 & 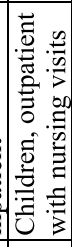 & $\begin{array}{l}\frac{5}{0} \\
\frac{7}{0} \\
\frac{7}{0} \\
\frac{1}{3} \\
\frac{5}{2}\end{array}$ & 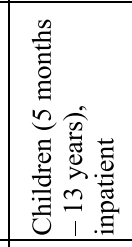 & 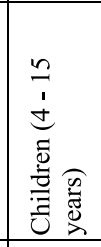 & 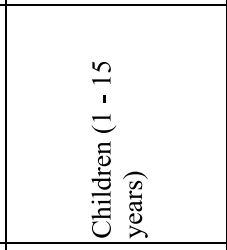 & 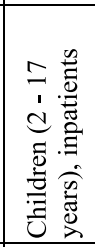 & 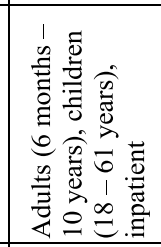 & 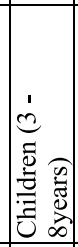 & 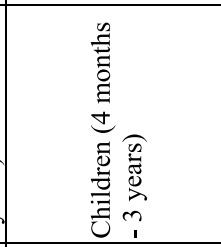 \\
\hline $\begin{array}{l}\text { छ } \\
\text { है }\end{array}$ & i & $\bar{v}$ & 0 & $\bar{m}$ & $\stackrel{ }{1}$ & 우 & ¿ & $\stackrel{\sim}{\sim}$ & $\infty$ & $\stackrel{2}{2}$ \\
\hline 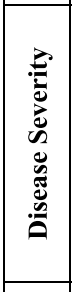 & 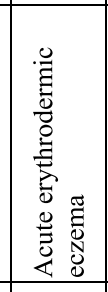 & 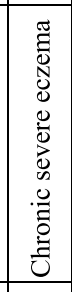 & 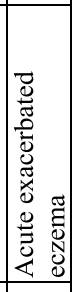 & 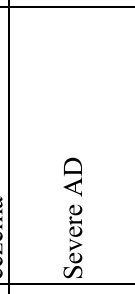 & 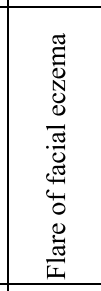 & 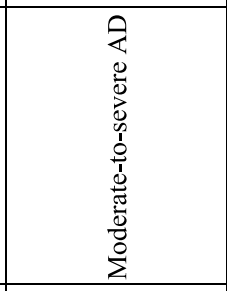 & 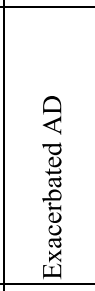 & 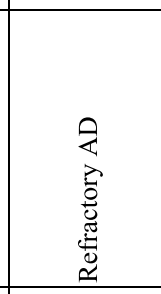 & 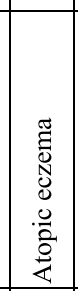 & 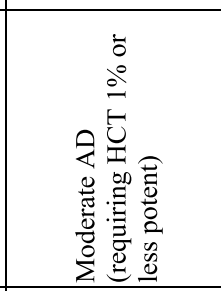 \\
\hline 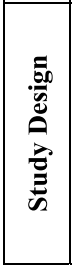 & 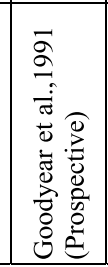 & 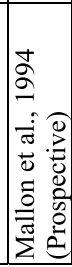 & 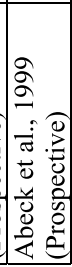 & 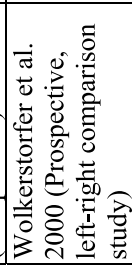 & 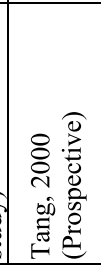 & 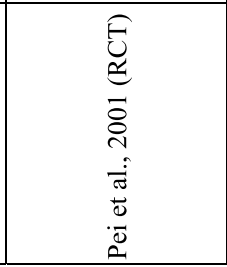 & 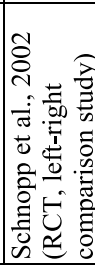 & 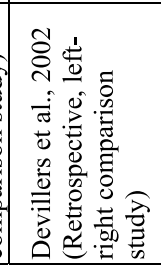 & 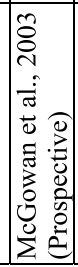 & 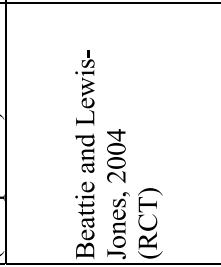 \\
\hline
\end{tabular}




\begin{tabular}{|c|c|c|c|c|c|c|c|c|c|}
\hline 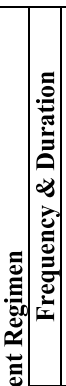 & 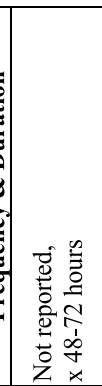 & 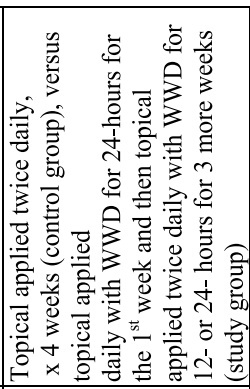 & 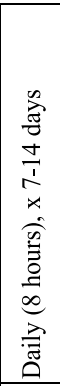 & 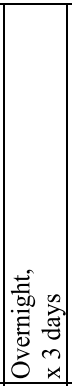 & 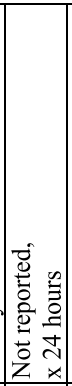 & 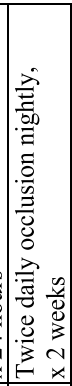 & 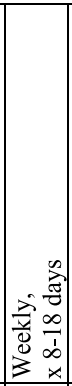 & 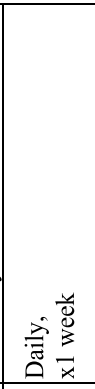 & 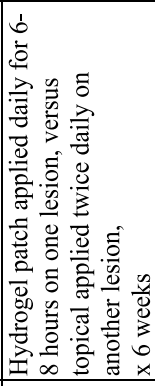 \\
\hline 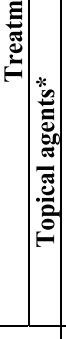 & 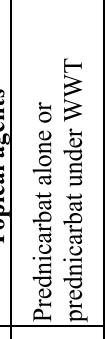 & $\stackrel{\circ}{\stackrel{0}{0}}$ & 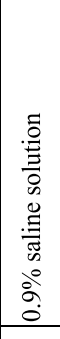 & 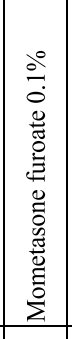 & $\begin{array}{l}0 \\
: \\
\check{0} \\
z\end{array}$ & 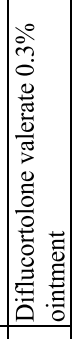 & 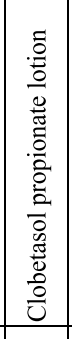 & 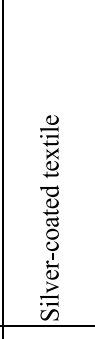 & 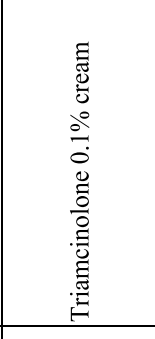 \\
\hline 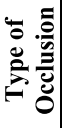 & 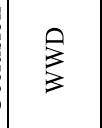 & $\sum_{3}^{2}$ & 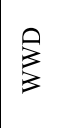 & 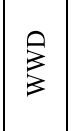 & 拿 & 会 & 商 & 高 & 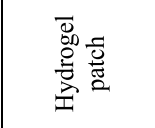 \\
\hline 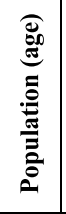 & 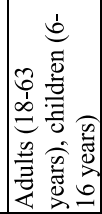 & 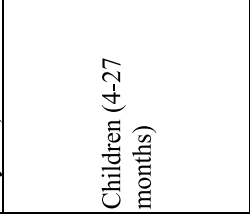 & 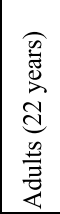 & 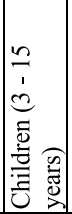 & 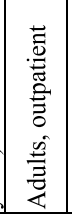 & 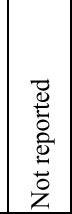 & $\frac{0}{3}$ & 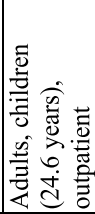 & 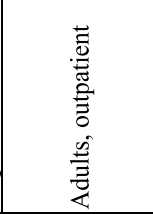 \\
\hline $\begin{array}{l}\widehat{\Xi} \\
\text { ஸै }\end{array}$ & $\stackrel{\sim}{\sim}$ & in & 으 & 0 & 으 & 8 & $\stackrel{\infty}{+}$ & $\cong$ & $\because$ \\
\hline 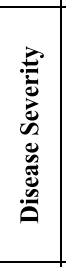 & 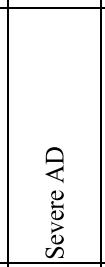 & 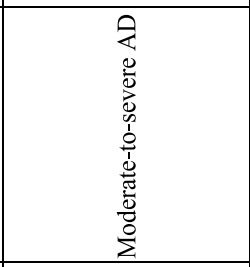 & 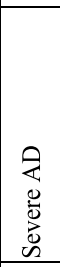 & 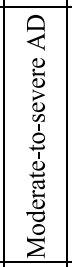 & 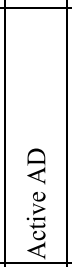 & 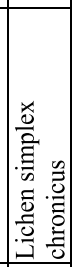 & 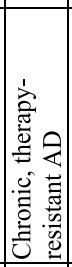 & 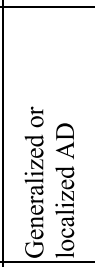 & 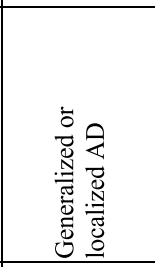 \\
\hline 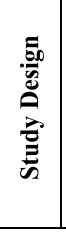 & 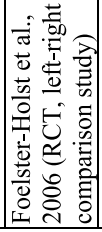 & 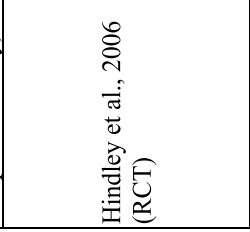 & 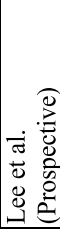 & 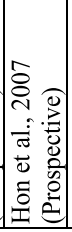 & 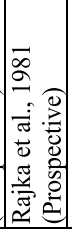 & 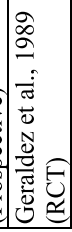 & 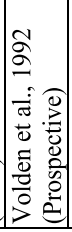 & 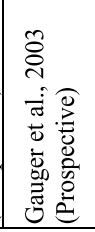 & 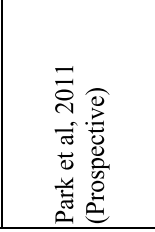 \\
\hline
\end{tabular}

*Dilution and concentration noted when provided in the article

Table 1. Studies on Occlusive Therapy $(\mathrm{HCT}=$ Hydrocortisone, $\mathrm{RCT}=$ randomized control trial) 
occlusion studies demonstrated efficacy in severe or acute, moderate and chronic AD. Among the 4 studies examining DWD, only one used a randomized controlled design. All, except one, DWD studies demonstrated improvements in AD. There is also one study that used an impermeable hydrogel patch consisting of about $50 \%$ water content. The hydrogel patch alone demonstrated improvements in $\mathrm{AD}$, comparable to that of corticosteroid use alone. Increased cutaneous bacterial counts or clinical infections were reported in 4 out of the 15 studies using WWD and all 4 studies using DWD.

\subsection{Wet wrap dressings}

The following is a detailed description of the 14 studies evaluating the efficacy of WWD in the treatment of AD (Table 2). Occlusive therapy was first introduced by Goodyear et al. in 1991. Goodyear et al. (1991) evaluated the inpatient use of WWD occlusion for acute erythrodermic eczema. This WWD technique involved the application of open-weave cotton tubular dressings (Tubegauz ${ }^{\circledR}$ ) impregnanted with hydrocortisone $1 \%$ cream (if child $<2$ years of age) or $10 \%$ dilution of bemethasone valerate (if child $>2$ years of age) twice daily. All 30 children responded well to WWD occlusion, with no relapses noted at 2 weeks follow-up. Interestingly, an attempt at long-term home therapy using WWD, following the inpatient therapy, in 5 patients was unsuccessful due to reports of inconvenience, increased bacterial infections, and prolonged HPA axis suppression. (Goodyear 1991)

Mallon et al. (1994) studied the use of WWD in chronic severe eczema in 21 children. Eczema was managed using topical steroid creams (i.e. hydrocortisone $0.5 \%$ or $10 \%$ dilution betamethosone $0.1 \%$ cream) and emollients under WWD daily for less than 5 days. All patients responded well to WWD therapy. The treatment was also well-tolerated. Majority of parents (20/21) reported decreased use topical steroid per week following the introduction of WWD therapy. (Mallon et al. 1994)

Abeck et al. (1999) treated 6 patients ( 3 children and 3 adults) with acute exacerbated atopic eczema with basic emollients in combination with chlorhexidine-soaked dressings for 3 days. The study observed improvements in disease severity based on SCORAD score. Patients also reported decreased itch and sleep loss following WWD therapy. In addition, there was a reduction of $S$. aureus counts that paralleled skin improvement. (Abeck et al. 1999)

In a study of 31 children with severe refractory AD, Wolkerstorfer et al. (2000) investigated the efficacy of various cortiocosteroid dilutions under WWD occlusion. Participants were divided into 3 treatment groups. The first group consisted of 18 children, who were treated with $50 \%$ dilution of fluticasone propionate $0.05 \%$ (FP) cream under WWD for 2 weeks. In the second group, 5 children with symmetrically localized AD were treated with different dilutions $(10 \%, 25 \%$ and $50 \%)$ of FP cream on the left and the right side of the body under WWD for one week and then $10 \%$ dilution of FP cream under WWD the following week. In the third group, 8 children were treated with $0 \%$ (i.e. only emollient), $5 \%, 10 \%$, or $25 \%$ dilutions of FP cream applied to the entire body under WWD. After just one week of therapy, significant improvement in disease severity was observed, without noticeable differences between $5 \%, 10 \%$, or $25 \%$ dilutions of FP cream under WWD. Less improvement was observed in the second week of therapy. In terms of skin infections, mild-to-moderate folliculitis was reported in a large proportion of the children, with $33 \%(6 / 18)$ children in the first group, $40 \%(2 / 5)$ children in the second group, and $63 \%(5 / 8)$ children in the third group. There was also one case of furunculosis in the third group. Interestingly, generalized folliculitis was noted in both of the children treated with only emollient during the first week. These findings suggests that although WWD occlusion may foster bacterial growth, 


\begin{tabular}{|c|c|c|c|c|c|c|c|c|c|c|}
\hline \multirow{3}{*}{ 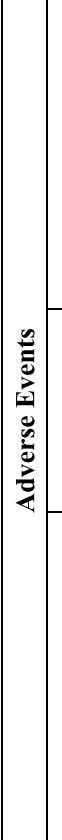 } & 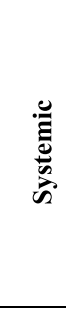 & 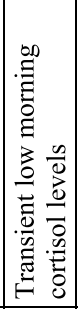 & $\underset{\mathrm{Z}}{\overleftrightarrow{L}}$ & $\underset{z}{\varangle}$ & 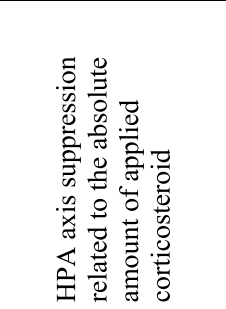 & $\frac{\varangle}{Z}$ & $\underset{z}{\varangle}$ & 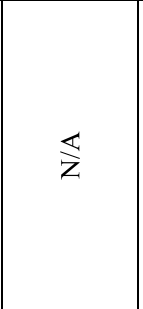 & 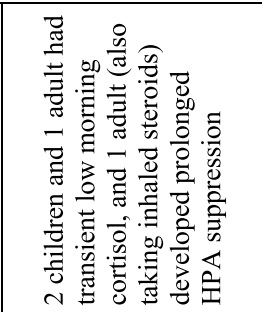 & $\frac{\varangle}{z}$ \\
\hline & 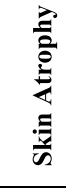 & 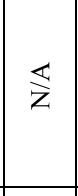 & 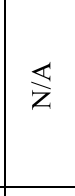 & $\overleftrightarrow{Z}$ & $\begin{array}{l}\text { İ } \\
\text { ż }\end{array}$ & $\begin{array}{l}\text { Z̃ } \\
\text { z }\end{array}$ & $\overleftrightarrow{Z}$ & $\overleftrightarrow{Z}$ & 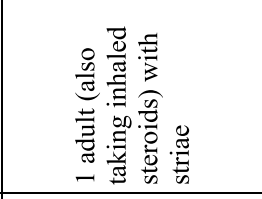 & 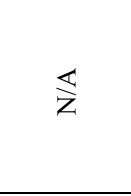 \\
\hline & & $\overleftrightarrow{\mathrm{z}}$ & $\begin{array}{l}\text { Ẽ } \\
\text { ż }\end{array}$ & 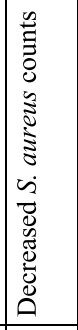 & 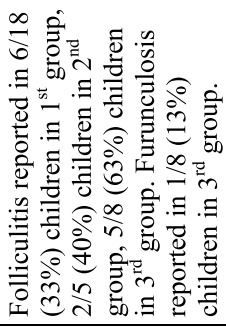 & $\begin{array}{l}\text { Z̃ } \\
\text { z }\end{array}$ & 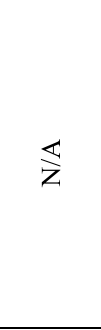 & 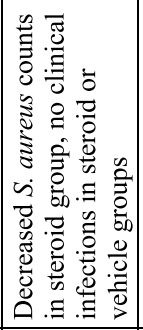 & 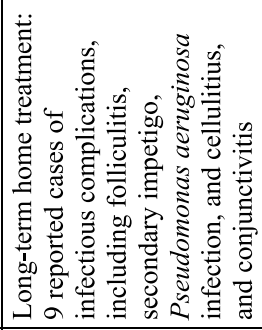 & $\underset{z}{\ll}$ \\
\hline \multirow{2}{*}{ 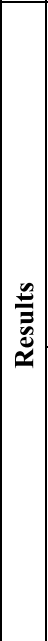 } & 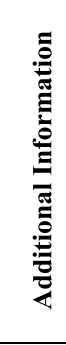 & 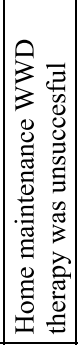 & 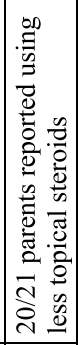 & 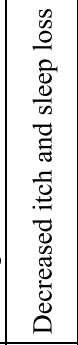 & 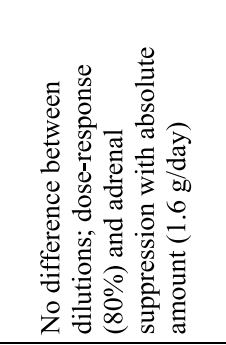 & & 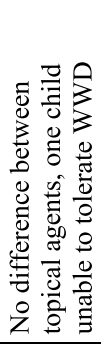 & 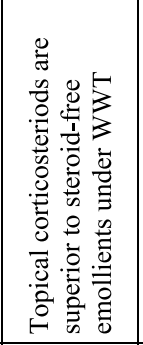 & 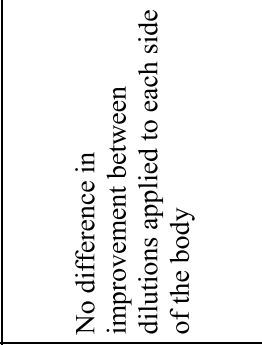 & 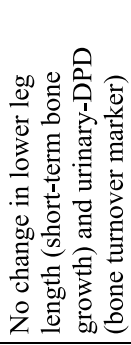 \\
\hline & 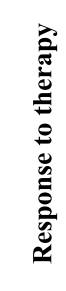 & 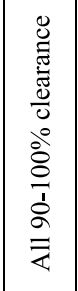 & 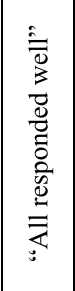 & $\begin{array}{l}\text { 离 } \\
0 \\
0 \\
0 \\
0 \\
0 \\
0 \\
0 \\
\text { : }\end{array}$ & 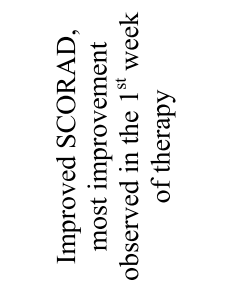 & 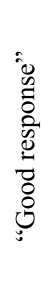 & 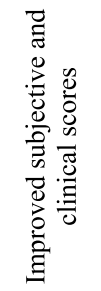 & 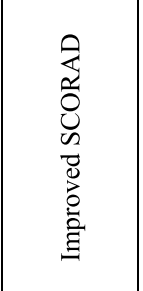 & 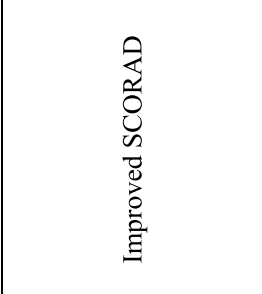 & $\underset{z}{\varangle}$ \\
\hline & 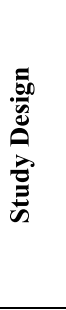 & 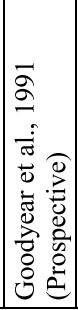 & 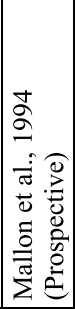 & 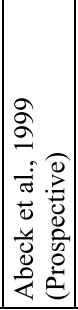 & 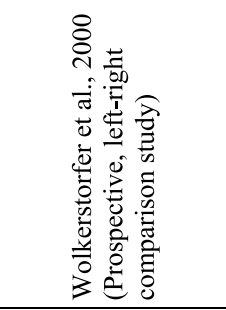 & 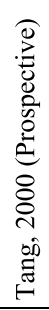 & 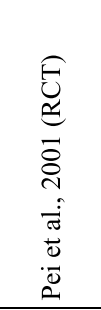 & 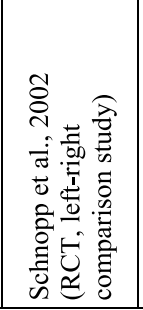 & 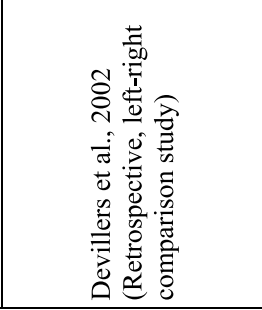 & 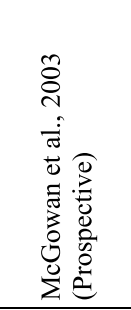 \\
\hline
\end{tabular}




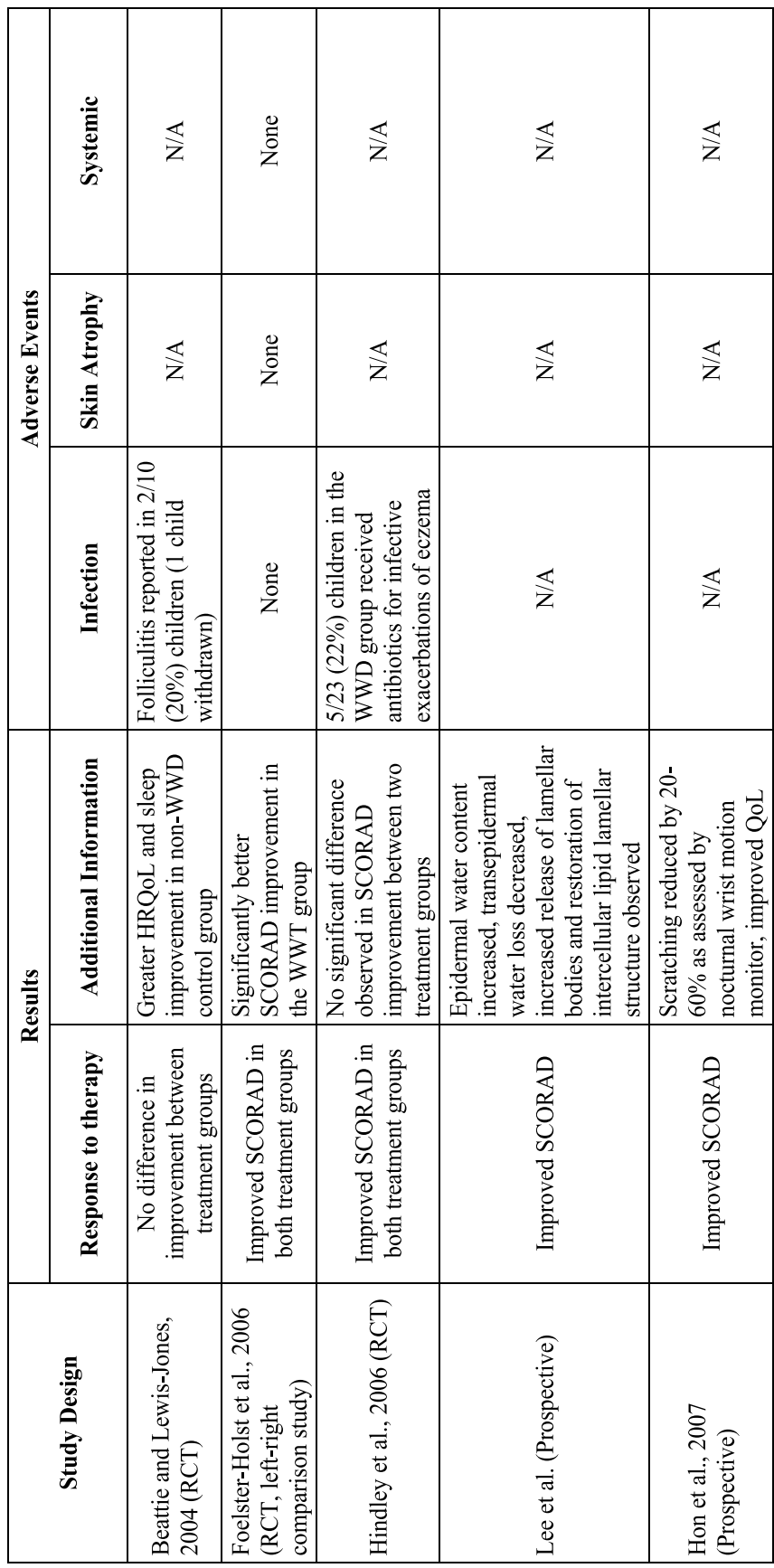

Table 2. Outcomes of Studies on Wet Wrap Dressing Occlusion (DPD = deoxypyridinoline, $\mathrm{HPA}=$ hypothalamic-pituitary-adrenal, HRQoL = Health Related Quality of Life) 
the addition of topical corticosteroids to the treatment regimen appeared to provide some protective benefits. (Wolkerstorfer et al. 2000)

Tang (2000) treated 10 children experiencing a flare of facial eczema with WWD occlusion. Children were initiated on an intermittent treatment regimen involving $10 \%$ dilution of mometasone furoate $0.1 \%$ cream or ointment under WWD applied for 2-3 hours once daily for a few consecutive days. All parents reported good treatment response. No cutaneous side effects were observed from topical corticosteroid use. (Tang 2000)

Pei et al. (2001) performed a randomized control trial of 40 children with moderate-to-severe AD. Prior to starting the study, all patient were instructed to apply $0.005 \%$ flucinolone acetonide cream twice daily for 2 weeks to standardize treatment medications. Patient were then randomized to receive $10 \%$ dilution of $0.1 \%$ mometasone furoate ointment or $10 \%$ dilution of $0.005 \%$ fluticasone propionate ointment. These topical agents were applied once a day for 2 weeks without WWD. After this 2-week period of open application, patients were further randomized to receive the same topical agent for 2 more weeks without WWD, or for 2 more weeks under WWD. Only 30 patients (75\%) entered into this second phase of the study since their disease severity had failed to improve by more than $50 \%$ after the initial 2 weeks of open topical application. In other words, only patients whose disease was refractory to a 2-week period of conventional open therapy continued in the study. Ultimately, there were a total of 27 patients who completed the study. One child receiving fluticasone propionate ointment dropped out of the study because the patient was unable to tolerate WWD. The study found that significantly greater improvements in disease severity and extent in patients using WWD, as compared to controls. These results suggest that WWD may be an effective second-line therapy in children whose disease is refractory to conventional open topical corticosteroids. (Pei et al. 2001)

Schnopp et al. (2002) examined the effect of WWD in a randomized control study of 20 inpatients with exacerbated AD. Children received either a topical corticosteroid preparation (mometasone furoate $0.1 \%$ ) or a steroid-free preparation (its vehicle) under WWD, applied twice daily for 5 days to the tested area in a left-right study. Disease severity at day 3 and day 5 continuously improved in both groups; however, the mometasone furoate-treated group showed significantly greater irmprovements. Staphylococcus aureus bacterial counts initially decreased during the first 3 days of treatment in both groups. At day 5, bacterial counts continued to decrease in the steroid-treated group, but bacterial counts increased in the vehicle-treated group. There were no reported clinical signs of bacterial superinfection in either the steroid or vehicle groups. The study concluded that WWD were useful in treatment of exacerbated AD, with applications of topical corticosteroids showing better efficacy than steroid-free emollients. (Schnopp et al. 2002)

Devillers et al. (2002) evaluated the use of WWD in refractory AD in 12 adults and 14 children. WWD were applied daily and re-wettted every 2-3 hours for 1 week in an inpatient setting. Patients used 5\% dilution of FP cream under WWD on affected areas of the face. As for the body, a side-to-side comparison study was performed, in which adults applied $10 \%$ and $25 \%$ dilutions of FP cream and children applied $5 \%$ and $10 \%$ dilutions of FP cream. Disease severity improved dramatically during the one week of inpatient therapy. There was no difference in improvement between dilutions applied to each side of the body. Following inpatient therapy, 8 adults and 13 children continued treatment at home with less potent dilutions of FP cream. Exacerbation of $\mathrm{AD}$ occurred in 3 adults and 2 children. Infectious complications included localized folliculitis (4 reported cases), secondary impetigo (2 reported cases), localized Pseudomonas aeruginosa infection, cellulitis of the left 
cheek in a patient without a facial mask, and purulent conjunctivitis in a patient with a facial mask. These complications may be attributed to variations in standard protocal for WWD application, such as prolonged ( $>8$ hours) occlusion and the frequent rewetting procedure. (Devillers et al. 2002)

McGowan et al. (2003) examined the effects of WWD therapy using topical corticosteroids on short-term growth and bone turnover in 8 prepubertal children, ranging from 3-8 years of age. Tubular bandages were applied over $10 \%$ or $25 \%$ dilutions of beclomethasone dipropionate for 24 hours each day for 2 weeks. After 2 weeks, frequency of tubular bandages was reduced to overnight use for one week and then as required for the remainder of the treatment period. Occlusive dressings were applied for a median duration of 12 weeks (range 2 - 18 weeks). Short-term growth was assessed by measuring lower leg length velocity by knemometry, while bone turnover was assessed by urinary deoxypyridinoline excretion. (McGowan et al. 2003) (See Section 3.4 Cutaneous and Systemic Side Effects for further discussion of this study)

In randomized control trial, Beattie and Lewis-Jones (2004) compared the use of $1 \%$ hydrocortisone under WWD to conventional open therapy in 19 children with moderate, widespread AD. The control group applied 1\% hydrocortisone twice daily for 2 weeks, without WWD. In the study group, patients applied 1\% hydrocortisone once in the morning for 2 weeks, with WWD twice daily for the first week and then only at night for the second week. Both groups were allowed to apply non-steroidal emollients as often as necessary. Beattie and Lewis Jones (2004) found no difference in clinical improvement between the control group and the study group. The authors, therefore, concluded that conventional open therapy using $1 \%$ hydrocortisone and emollients alone appeared to be as effective as using $1 \%$ hydrocortisone under WWD for moderate AD. Despite these findings, it is important to note that the use of $1 \%$ hydrocortisone is a less potent choice than what is generally used in clinical practice when treating moderate AD. If the authors had evaluated the use of a mid-potency topical corticosteroid under WWD, the degree of improvement between the study and control groups might have been markedly different. In terms of health related quality of life, Beattie and Lewis-Jones (2004) found greater improvements in the non-WWD control group than the WWD group for both the child and the family. Children in the non-WWD control group also reported more sleep than the WWD group, but there was no significant difference for itch between treatment groups. In regards to infections, 2 of the $10(20 \%)$ children in the WWD group experienced folliculitis, one of which had to be withdrawal from the study. There were no reported clinical infections in the non-WWD control group. (Beattie and Lewis-Jones 2004)

Foelster-Holst et al. (2006) conducted a randomized, controlled study of 24 adults and children with acute episodes of AD. This was left-right comparison study, in which patients had skin lesions symmetrically affecting both arms or legs. One arm or leg was randomly treated with the topical prednicarbat (a medium potency corticosteroid), with WWD using a tubular bandage. The other extremity received topical prednicarbat alone. After 48-72 hours of therapy, both groups showed improvement of the local SCORAD, but the improvement in the WWD group was significantly better. No adverse effects were observed in either treatment group. (Foelster-Holst et al. 2006)

Hindley et al. (2006) carried a randomized, control trial to investigate the efficacy of WWD as compared to conventional topically applied corticosteroids. This 4-week study consisted of a total of 50 children with moderate-to-severe eczema. In the conventional treatment group, patients had emollients applied as needed and 1\% hydrocortisone ointment (or more 
potent topical steroids, if necessary) applied twice daily. In the WWD treatment group, patients had wet wraps applied daily for a 24 -hour period over $1 \%$ hydrocortisone ointment (or more potent topical steroids, if necessary) during the first week, followed by wet wraps applied for a 12- or 24-hour period depending on disease progress for the remaining three weeks. When wet wraps were applied for only a 12-hour period, $1 \%$ hydrocortisone and emollients were used during the non-wet wrap period. Both treatment groups demonstrated improvement in overall SCORAD scores; however, there was no significant difference between the conventional and WWD treatment groups. Five out of $23(22 \%)$ children in the WWD treatment group required antibiotics for skin infections, as compared to none of the children in the conventional treatment group. The authors conclude that 4-weeks of maintenance WWD treatment may be associated with more skin infections than conventional treatment. (Hindley et al. 2006) However, it is important to realize that in clinical practice, physicians usually do not use WWD for maintenance therapy of AD over a 4-week duration. Instead, physicians are more likely to use WWD in settings of acute generalized eczematous flares for 3-7 days to induce disease remission. (Williams 2006) Lee et al. (2007) examined the therapeutic efficacy of WWD and the mechanism behind its therapeutic efficacy in treatment of AD. Ten patients with severe AD received WWD (without steroid treatment) for 7-14 days. SCORAD was used to assess AD severity, immediately following the end of treatment and 7 days after termination of treatment. Transepidermal water loss, water content in the corneum, and lipid amount of skin surface were also measured. The SCORAD was significantly reduced after WWD therapy. Additionally, epidermal water content was increased and transepidermal water loss was decreased following WWD therapy; these results were maintained 1 week after terminating therapy. In atopic lesions, increased release of lamellar bodies and restoration of intercellular lipid lamellar structure was observed. The authors speculated that increased secretion of lamellar bodies induced with WWD occlusion may lead to recovery of the abnormal epidermal barrier and clinical improvement in AD. (Lee et al. 2007)

In a clinical study of six children with moderate to severe AD, Hon et al. (2007) tested the efficiacy of WWD occlusion using Tubifast ${ }^{\circledR}$ garments with mometasone furoate $0.1 \%$ cream. Short-term use of WWD occlusion over 3 days demonstrated improvement in disease severity based on SCORAD. In addition, a wrist motion monitor was used to measure nocturnal itch, which showed that average scratching activity was significantly reduced by $20-60 \%$ on day 3 of treatment. Furthermore, WWD was effective in improving quality of life in these children. (Hon et al. 2007)

Since its introduction by Goodyear et al. (1991) nearly two decades ago, WWD occlusion has been extensively used as a relatively safe and effective treatment modality for children with acute erythrodermic $\mathrm{AD}$ and those with severe and/or refractory $\mathrm{AD}$. The most effective topical corticosteroid to be used is still uncertain, but $10 \%$ dilutions of potent topical corcorticosteroid are most commonly used. Protocols on adminstration and duration of WWD occlusion can vary between studies. (Oranje et al. 2006) (See Table 3)

Advantages of WWD occlusion include a rapid therapeutic response, reduction in itch and sleep disturbances, and a possible decreased topical corticosteroid use. Disadvantages include high cost, the need for specialized training, increased potential for topical corticosteroid absorption, and increased incidences of folliculitis and other cutaneous infections. (Oranje et al. 2006)

However, the application of WWD has become less time-consuming and more feasible for home use with development of Tubifast ${ }^{\circledR}$ garments manufactured by Medlock Medical ${ }^{\circledR}$ 
(available since 2003). With the introduction of these garments, wet wrapping can be done in 20-25 minutes. Tubifast garments ${ }^{\circledR}$ are available as tights for babies aged 6-24 months, vests for children 6 months through 14 years, and socks and leggings in all sizes. These washable garments are absorbent, holding enough water to remain moist for hours, and are elastic and able to conform to the contours of the body. (Page 2005)

1. Choose the appropriate width of the tubular bandages and cut these to size to fit the affected body areas (i.e. arms, legs, trunk). Alternatively, Tubifast ${ }^{\circledR}$ garments can be used.

2. Apply the appropriate dilution of topical corticosteroids (e.g. fluticasone propionate $0.05 \%$ cream, mometasone furoate $0.1 \% \mathrm{cream}$ ) on the involved skin. In general, diluted steroids in emollients of 1:9 are applied to the face of all patients and body of infants, while diluted steroids in emollients of 1:9 or 1:3 are applied on the body of adults.

3. Wet the pieces of tubular bandage in lukewarm water. Alternatively, if Tubifast ${ }^{\circledR}$ garments are used, then the inner garment is moistened using a plant sprayer.

4. Apply the first layer of wet tubular bandages. Connect the arm and leg pieces to the trunk. Apply the facial mask, if necessary. Alternatively, if Tubifast $\AA$ garments are used, the inner garment is moistened using a plant spray.

5. Apply the second layer of dry tubular bandage. Again, connect the arm and leg pieces to the trunk. Apply the facial mask, if necessary. Alternatively, if Tubifast ${ }^{\circledR}$ garments are used, a second dry Tubifast ${ }^{\circledR}$ garment is placed over the wet one.

6. Re-wet the tubular bandages every 2-3 hours. Alternatively, if Tubifast ${ }^{\circledR}$ garments are used, re-wet the inner Tubifast ${ }^{\circledR}$ garment every 2-3 hours.

7. Repeat procedures \#1-6 (described above) daily.

8. After 7 days of occlusive therapy, the dilution of topical corticosteroids (e.g. fluticasone propionate $0.05 \%$ cream, mometasone furoate $0.1 \%$ cream) is applied on the involved skin for 4-7 consecutive days.

Table 3. Protocol for Wet Wrap Dressing occlusion

Finally, in a review article, Devillers and Oranje (2006) made the following conclusions regarding WWD occlusion with a grade $C$ of recommendation. 1) WWD using cream or ointment and a double layer of cotton bandages, with a moist first layer and a dry second layer, is an efficacious short-term treatment for children with severe and/or refractory AD.2) The use of WWD with diluted topical corticosteroids is a more efficacious treatment than wet-wrap dressings with emollients only for children with severe and/or refractory AD. 3) The use of WWD with diluted topical corticosteroids is a safe intervention treatment for children with severe and/or refractory $\mathrm{AD}$ for up to 14 days, with temporary systemic bioactivity of the corticosteroids as the only reported serious adverse effect. 4) Lowering the absolute amount of applied topical corticosteroid to once daily application and further dilution can reduce potential risks. (Devillers and Oranje 2006)

\subsection{Dry wrap dressings}

DWD occlusion in AD has been less well studied (Table 4). Specifically, Rajka et al. (1981) examined the effect of dry occlusion on skin microbial flora. Occlusion using plastic film was applied to 10 patients with AD. A significant increase in the density of $S$. aureus was observed after 24 hours of dry occlusion. Notably, 2 of the 10 patients had tiny pustules or crusts following dry occlusion. But, there were no reports of AD exacerbation. (Rajka et al. 1981) 


\begin{tabular}{|c|c|c|c|c|c|}
\hline \multirow{3}{*}{ 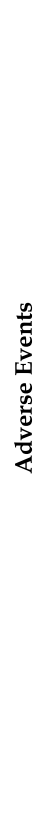 } & 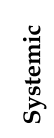 & $\frac{\varangle}{\bar{z}}$ & $\frac{\mathbb{Z}}{\mathrm{z}}$ & $\frac{\ll}{z}$ & $\frac{\varangle}{z}$ \\
\hline & 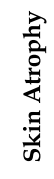 & $\frac{\varangle}{\mathrm{z}}$ & $\frac{\varangle}{\mathrm{z}}$ & $\begin{array}{l}\text { ஜ̆ } \\
\text { Zे }\end{array}$ & $\frac{\varangle}{\mathrm{z}}$ \\
\hline & 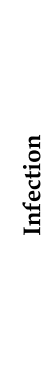 & 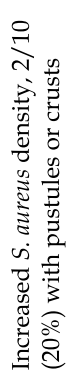 & 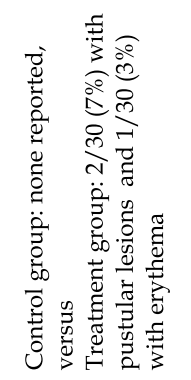 & 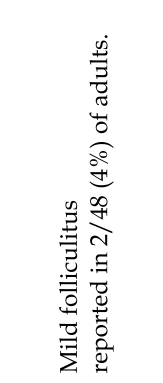 & 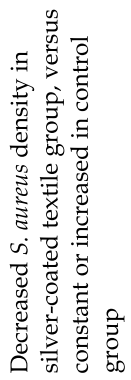 \\
\hline \multirow{2}{*}{ 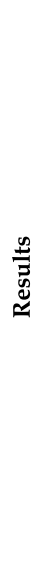 } & 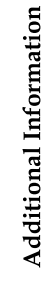 & 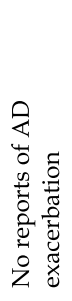 & & 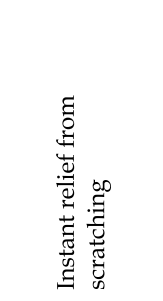 & \\
\hline & 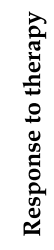 & $\frac{\mathbb{z}}{\mathrm{z}}$ & 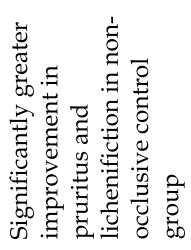 & 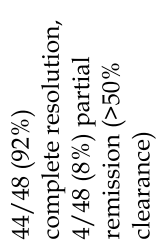 & 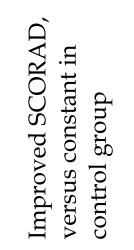 \\
\hline & مُ & 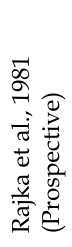 & 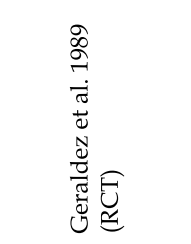 & 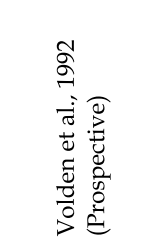 & 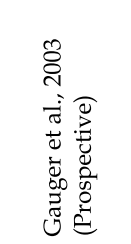 \\
\hline
\end{tabular}

Table 4. Outcomes of Studies on Dry Wrap Dressing Occlusion 
Geraldez et al. (1989), in a randomized controlled study of 60 patients with lichen simplex chronicus, observed more infections and other adverse events in patients treated with diflucortolone valerate $0.3 \%$ ointment under occlusion as compared those treated without occlusion. Among the patients using dry occlusion, 2 patients $(7 \%)$ developed pustular lesions surrounding the affected sites, one (3\%) had erythema, and one $(3 \%)$ had hyperpigmentation. None in the control group experienced any side effects. The control group also reported greater improvements in both pruritus and lichenication. The authors noted that the difference in the observed efficacy may be due to poorer absorption of the ointment that tended to adhere to the plastic occlusive material. Furthermore, the adverse events seen in the treatment group may have resulted from the combination of both the ointment vehicle and the nature of the plastic occlusive material that created an overocclusive environment, thereby favoring microbial proliferation and/or contact sensitization. (Geraldez et al. 1989)

Volden et al. (1992) treated 48 patients with chronic, therapy-resistant AD, with once-weekly application of clobetasol propionate lotion under hydrocolloid (Duoderm ${ }^{\circledR}$ ) occlusive dressings. Of the 48 patients, $44(92 \%)$ had complete resolution of lesions, and $4(8 \%)$ had partial response (defined as $>50 \%$ clearance). There were also $2(4 \%)$ patients with mild folliculitis. The authors conclude that adverse events were both mild and infrequent. (Volden 1992)

Gauger et al. (2003) further addressed the concern of skin colonization with S. aureus using occlusion therapy for AD. A side-to-side comparative study of 15 patients with generalized or localized $\mathrm{AD}$ was performed, in which the flexures of the elbows were covered with silver-coated textiles on one arm and cotton on the other arm for one week. The study found a significant decrease in $S$. aureus colonization on the lesions covered with silver-coated textile just 2 days after initiation of therapy and lasting until the end of the treatment. Additional, a significantly less amount of $S$. aureus was observed on the silver-coated textile sites than the cotton sites at the end of the treatment. Clinical improvement further correlated with the reduction of bacterial colonization. Thus, the silver-coated textile appeared to not only improve active lesions of $\mathrm{AD}$, but also mitigate the potential promicrobial effects of dry occlusion alone. (Gauger et al. 2003)

Ultimately, DWD occlusion appears to improve disease severity and is perhaps most beneficial in chronic treatment-resistant eczematous lesions. However, there is too little data to determine whether occlusion, in particular DWD occlusion, predisposes skin bacterial growth. But, it seems reasonable to conclude that the addition of antimicrobial topical agents (e.g. antiseptic or silver preparations) to occlusive therapy might be helpful in countering the potential risk of infection. (Abeck et al. 1999; Gauger 2006)

\subsection{Hydrogel patch}

In a pilot study of 15 patients, Park et al. (2011) evaluated the efficacy and safety of a hydrogel patch for AD treatment. The hydrogel patch used in this study was composed of an adhesive, thin, flexible, hydrogel layer on an impermeable urethane surface. Unlike hydrocolloid dressings (e.g. Duoderm ${ }^{\circledR}$ ) with low water content, the hydrogel patch consisted of approximately $50 \%$ water. In this 6-week study, patients applied the hydrogel patch over one lesion for 6-8 hours daily and triamcinolone (TAC) $0.1 \%$ cream twice daily to another lesion. Erythema, induration, lichenification, excoriation, and total EASI scores significantly improved compared to baseline in both the hydrogel patch and TAC groups. 
Improvement in pruritis was observed in both treatment groups, but was only statistically significant in the TAC group. At week 4, there was no significant difference in all subscores between the patch and TAC groups (except pruritus, as stated above). Improvement was maintained after discontinuing treatment for 2 weeks. No adverse events from steroid use occurred. This study appears to demonstrate that instant correction of the dysfunctional skin barrier with the hydrogel patch can improve signs and symptoms of AD comparable to TAC $0.1 \%$ cream. Thus, the hydrogel patch may provide a new approach to occlusive therapy without the potential risks of topical corticosteroid use (Park et al. 2011)

\subsection{Cutaneous and systemic side effects}

Topical corticosteroid use can cause both cutaneous and systemic side effects, most significantly laboratory adrenal insufficiency. Risk factors include the use of high potency corticosteroids, occlusive or prolonged therapy, and application to thin- or barriercompromised skin lesions. (Levin and Maibach 2002) Occlusive therapy with topical corticosteroids does not appear to be associated with an increased incidence of local noninfectious side effects, such as skin atrophy and striae. This is most likely because nearly all studies have examined limited treatment durations and diluted topical corticosteroids. However, several studies demonstrated either lowering of morning cortisol levels (Hartmann and Lahmann 1977; Goodyear et al. 1991), loss of diurnal cortisol rhythm (Hartmann and Lahmann 1977), or overt laboratory HPA suppression with WWD (Wolkerstorfer et al. 2000; Devillers et al. 2002). Importantly, nearly all cases of decreased cortisol levels proved to be transient, returning to normal ranges within weeks of discontinuing therapy.

Specifically, Goodyear et al. (1991) found that 0900 hour cortisol levels were suppressed immediately after treatment with WWD, but returned to normal 2 weeks following therapy. Following this observation by Goodyear et al. (1991) of a transient lowering of morning cortisol levels (Goodyear et al. 1991), Wolkerstorfer et al. (2000) decided to examine the effects of different corticosteroid dilutions under WWD occlusion on HPA axis supression in children with severe refractory AD. Only 3 out of the 18 children in the first treatment group (i.e. $50 \%$ dilution of FP cream) and none of the 5 children in the second treatment group (i.e. $10 \%, 25 \%$ and $50 \%$ dilutions of FP cream) demonstrated HPA axis suppression based on 0900 hour serum cortisol measurements following 2 weeks of WWD occlusion. In the third treatment group (i.e. $0 \%, 5 \%, 10 \%$, or $25 \%$ dilutions of FP cream applied to the whole body), children demonstrated HPA axis suppression based on 0600 hour serum cortisol measurements, which was associated with the absolute amount of applied corticosteroid. To be more specific, a dose-response relationship was observed with an absolute amount of topical corticosteroid applied. Patients using $800 \mu \mathrm{g} \mathrm{m}^{-2}$ daily (equivalent to $1.6 \mathrm{~g}$ FP $0.05 \%$ cream) had an approximately $80 \%$ improvement, while patients using absolute amounts $>957 \mu \mathrm{g} \mathrm{m}^{-2}$ daily had no greater improvement. Patients using absolute amounts $\leq 800 \mu \mathrm{g} \mathrm{m}^{-2}$ daily had no suppression of the HPA axis. This suggests that weaker dilutions of topical corticosteroid may have a lower risk of HPA axis suppression, while still maintaining comparable efficacy to more potent dilutions of topical corticosteroid. (Wolkerstorfer et al. 2000)

Similarly, Devillers et al. (2002) found a significant decrease in early-morning serum cortisol levels following 1-week of inpatient WWD therapy. Transient cortisol levels below the normal range were observed after 4 days in 3 children and after one week in 2 adults. One 
adult, who also taking inhaled steroids, developed prolonged suppression of the HPA axis, in combination with several abdominal striae. (Devillers et al. 2002)

As for the effect of occlusive therapy using topical corticosteroids on bone development, McGowan et al. (2003) examined short-term growth and bone turnover in prepubertal children undergoing WWD occlusion for AD. Knemometry, a technique estimating the distance between the heel and knee of the sitting child, was used as a non-invasive measurement of lower leg length, in combination with 24-hour urinary deoxypyridinoline excretion to evaluate bone turnover. Lower leg length and urinary deoxypyridinoline levels for all children remained similar in pre-treatment measurements and during therapy. This suggests that WWD occlusion for a limited duration does not impact growth parameters. (McGowan et al. 2003)

\section{Occlusion in clinical practice}

Occlusive therapy, in particular WWD occlusion, can be useful as a 'stepped-up' therapy in controlling acute erythrodermic $\mathrm{AD}$ or as a second-line therapy for severe and/or refractory disease. There is limited convincing data that WWD occlusion is superior to conventional open application of topical corticosteroids. However, occlusive therapy represents an important alternative to currently available, but often undesirable standard treatment modalities for AD. A few drawbacks of standard therapies include dependency on superpotent topical corticosteroids, frequent and inconvenient sessions of phototherapy, and serious systemic side effects with oral medications (e.g. nephrotoxicity with cyclosporine use).

Based on the studies on occlusive therapy, it appears that the greatest benefits of topical corticosteroids under occlusion is achieved during the first week of therapy. This suggests that if used as 'rescue' therapy for acute flares or intermittently for maintenance, durations of up to 1 week might be adequate (Wolkerstorfer et al. 2000; Pei et al. 2001). Anecdotally, long-term effects are thought to be sustained when WWD occlusion are continued with emollients for 2-4 more weeks to improve skin hydration (Nicol 1987). Variations in protocol regarding duration of WWD occlusion and rewetting procedures possibly contributed to differences observed in efficacy and incidence of infection. It may, therefore, be advisable to limit occlusion to less than 8 hours duration, apply WWD no more than twice daily, and avoid rewetting procedures.

It is difficult to ascertain the extent to which occlusion may promote bacterial colonization. However, the use of anti-bacterial agents, when applied alone or under occlusion, appears to not only inhibit bacterial colonization, but also reduce disease severity. (Abeck et al. 1999; Brockow et al. 1999; Gauger 2006) Thus, it seems logical that antibacterials, whether topical or systemic, might be a beneficial adjunct to occlusive therapy helping to decrease the potential risk of clinical infection. As such, topical antibacterials in combination with topical corticosteroids under occlusion may be useful when treating small areas of skin for a limited period of time, while systemic antibacterials in combination with topical corticosteroid under occlusion may more be appropriate when treating larger areas of involvement. Yet, the use of antibacterials must be weighed against the potential risk of antimicrobial resistance and contact sensitization. (Williams 2000; Zhai and Maibach 2001)

More specifically, according to Williams (2000), there are three categories of patients with AD: 1) those with obvious clinical infection, in whom anti-staphylococcal therapy is essential, 2) those with mild disease and a lower density of $S$. aureus colonization, in whom no evidence supports additional benefit of anti-staphylococcal therapy, and 3) those with fissured or 
cracked eczema without overt signs of infection such as crusting or folliculitis. (Williams 2000) While the latter group of patients carry an intermediate to high levels of $S$. aureus, studies are contradictory regarding the clinical benefits of anti-staphylococcal therapy in these patients. Clinicians may choose to institute antimicrobial therapy, in combination with topical corticosteroids under occlusion. If poorly responsive to WWD occlusion, addition of corticosteroid with topical or oral antibiotics has been helpful anecdotally, suggesting that subclinical infection or heavy colonization may impede response to topical corticosteroids. (Nicol 1987) Despite half a century of topical corticosteroid use in eczematous skin, the clinical criteria for the addition of a topical antimicrobial agents remains to be determined.

In terms of the concern for adrenal suppression with topical corticosteroids under occlusion, the minimal available data suggests that a decrease in cortisol levels can occur, but these effects are generally transient. The use of mid- or high-potency corticosteroid, an ointment vehicle, occlusion, and a disrupted skin barrier are all contributing factors that can increase the risk of systemic side effects including this possibilty of adrenal suppression. Of note, there has been only one suggested fatal case of adrenal insuffiency from topical corticosteroid use. However, the details of this case are limited. It is known that the patient was an 11-year old girl with generalized psoriasis being treated with betamethasone cream under occlusion for a prolonged durations. However, the concentration and the exact quantity of betamethasone cream applied, as well as the duration of therapy are unknown. (Levin and Maibach 2002)

Several strategies may be used to limit the potential for such complications. Possible options include using occlusive therapy only intermittently, reducing topical corticosteroid potency gradually as inflamed skin improves, and applying occlusion only once-daily. Studies have demonstrated that twice-daily application of topical corticosteroids to be no more effective than once-daily regimens and more prone to systemic side effects. (Lagos and Maibach 1998; Green et al. 2005) In addition, diluting topical corticosteroids may be helpful in reducing the risk of serious side effects. However, such preparations may alter the physiochemical properties of the agent and may not serve to reduce disease activity. (Haigh and Smith 1991; Gao and Li Wan Po 1994; Ohtani et al. 2002; Kizu et al. 2004) Furthermore, the application of a hydrogel patch, which appeared to have similar efficacy as topical corticosteroid use, may potentially serve as an ideal method of treating $\mathrm{AD}$ while avoiding topical corticosteroids. (Park et al. 2011) But, additional studies on the efficacy of the hydrogel patch in the treatment of $\mathrm{AD}$ are needed. Ultimately, if prolonged topical corticosteroid use is required in certain patients with refractory disease, adrenal function may be monitored using morning plasma cortisol and urinary steroid levels, in combination with rapid ACTH stimulation testing, while evaluating the patient for cutaneous atrophy or Cushingoid features (Levin and Maibach 2002). But, the rare occurence of topical corticosteroid-induced Addison's disease makes this suggestion impractical for most patients.

Topical immunosuppressive agents such as pimecrolimus or tacrolimus under occlusion have not yet been studied extensively. These agents might represent a valuable nonsteroidal alternative, though systemic absorption should be measured to confirm that immunosuppressive levels have not been reached. An open-label study evaluating topical pimecrolimus cream $1 \%$ in the treatment of chronic hand dermatitis showed that twice-daily application under occlusion was safe and effective, resulting in low pimecrolimus blood levels. (Thaci et al. 2003) Beyeler et al. (2006) reported a case of a female patient treated with tacrolimus $0.1 \%$ ointment under occlusion with Unna's paste boots (zinc bandages), whose 
serum levels had reached $12.9 \mu \mathrm{g} / 1$ after 5 days of treatment, corresponding to high-dose immunosuppression. Beyeler et al. (2006) attributed the increased systemic absorption to the combination of severely damaged skin barrier function, application of tacrolimus to a large surface area, and occlusion under zinc bandages. (Beyerler et al. 2006)

The wide variation in occlusive techniques and predominance of uncontrolled trials with small sample sizes make direct comparison of studies evaluating the safety and efficacy of occlusive therapy difficult. Limited numbers of studies using control groups restricts the conclusions that can be drawn regarding the benefit of occlusive therapy versus conventional therapies. However, it can be concluded that occlusive therapy has been shown, both formally and anecdotally, to be very effective in severe or exacerbated AD. It, therefore, offers an important alternative or adjunct therapy to more commonly used approaches. The randomized controlled trials described in this chapter resulted in divergent outcomes regarding infectious complications and efficacy. Differences could be related to the study populations (i.e. exacerbated versus moderate AD) or different topical corticosteroid application regimens.

While the exact mechanism of action of occlusive therapy is still unknown, a recent pilot study demonstrated significant down-regulation of 7 serum chemokines following WWD occlusion for 1 week in 6 children with severe AD. Of these, 4 chemokines are considered to be potential serum markers for the disease activity of AD. (Ong et al. 2008) In another study evaluating WWD occlusion using diluted tacrolimus and fluticasone propionate cream in APOC1 mice with AD, WWD occlusion improved transepidermal water loss, reinforcing that this may be one of the therapeutic aspects of occlusive therapy. (Oranje et al. 2009)

Future studies should evaluate the efficacy of occlusive therapy versus open topical corticosteroid application with measurements on the differences in systemic absorption. Studies should also investigate the impact of varying the durations of occlusive therapy. Given the number of protocols utilizing inpatient or specialized nurse-assisted visits, it would be useful to understand the contribution of professional supervised application. To our knowledge, no studies to date have evaluated occlusion as preventive therapy (i.e., to prevent disease flares and maintain cutaneous hydration).

\section{Conclusion}

WWD occlusion may be a reasonable first-line therapy for acute exacerbations of AD and second-line therapy for refractory disease. DWD occlusion appears to be efficacious having relatively low risk of infectious complications and may be particularly useful in treatment of chronic AD lesions. However, it remains unclear if occlusive therapy offers significant advantageous over conventional open therapy. The risk of infection associated with occlusive therapy, while greater than open therapies, seems to be manageable and may be mitigated by limiting the duration of occlusion, avoiding rewetting and perhaps adding antibacterial agents. Transient changes in morning cortisol levels and rare clinical HPA suppression seen with the use of topical corticosteroids under occlusion are concerning side effects, but are likely related to corticosteroid potency and the absolute amount applied. The hydrogel patch offers an innovative approach to the treatment of AD instantly correcting the barrier defect, and it may have similar efficacy as topical corticosteroids without the systemic risks. Further controlled studies are needed to better evaluate the risks and benefits of these occlusive modalities in the treatment of $\mathrm{AD}$. 


\section{References}

Abeck, D., K. Brockow, et al. (1999). Treatment of acute exacerbated atopic eczema with emollient-antiseptic preparations using the "wet wrap" ("wet pajama") technique. Hautarzt, 50(6): 418-421.

Aly, R., C. Shirley, et al. (1978). Effect of prolonged occlusion on the microbial flora, $\mathrm{pH}$, carbon dioxide and transepidermal water loss on human skin. I Invest Dermatol, 71(6): 378-381.

Arikawa, J., M. Ishibashi, et al. (2002). Decreased levels of sphingosine, a natural antimicrobial agent, may be associated with vulnerability of the stratum corneum from patients with atopic dermatitis to colonization by Staphylococcus aureus. J Invest Dermatol, 119(2): 433-439.

Beattie, P. E. and M. S. Lewis-Jones (2004). A pilot study on the use of wet wraps in infants with moderate atopic eczema. Clin Exp Dermatol, 29(4): 348-353.

Beyerler, M., P. Schmid-Grendelmeier, et al. (2006). Significantly elevated systemic levels after occlusive application of topical tacrolimus in atopic dermatitis. Dermatology, 212(3): 260-261.

Bridgman, A. (1995). The use of wet wrap dressings for eczema. Paediatr Nurs, 7(2): 24-27.

Brockow, K., P. Grabenhorst, et al. (1999). Effect of gentian violet, corticosteroid and tar preparations in Staphylococcus-aureus-colonized atopic eczema. Dermatology, 199(3): 231-236.

Devillers, A. C., F. B. de Waard-van der Spek, et al. (2002). Treatment of refractory atopic dermatitis using 'wet-wrap' dressings and diluted corticosteroids: results of standardized treatment in both children and adults. Dermatology, 204(1): 50-55.

Devillers, A. C. and A. P. Oranje (2006). Efficacy and safety of 'wet-wrap' dressings as an intervention treatment in children with severe and/or refractory atopic dermatitis: a critical review of the literature. Br J Dermatol, 154(4): 579-585.

Foelster-Holst, R., F. Nagel, et al. (2006). Efficacy of crisis intervention treatment with topical corticosteroid prednicarbat with and without partial wet-wrap dressing in atopic dermatitis. Dermatology, 212(1): 66-69.

Gao, H. Y. and A. Li Wan Po (1994). Topical formulations of fluocinolone acetonide. Are creams, gels and ointments bioequivalent and does dilution affect activity? Eur J Clin Pharmacol, 46(1): 71-75.

Gauger, A. (2006). Silver-coated textiles in the therapy of atopic eczema. Curr Probl Dermatol, 33: 152-164.

Gauger, A., M. Mempel, et al. (2003). Silver-coated textiles reduce Staphylococcus aureus colonization in patients with atopic eczema. Dermatology, 207(1): 15-21.

Geraldez, M. C., M. Carreon-Gavino, et al. (1989). Diflucortolone valerate ointment with and without occlusion in lichen simplex chronicus. Int J Dermatol, 28(9): 603-604.

Goodyear, H. M., K. Spowart, et al. (1991). Wet-wrap' dressings for the treatment of atopic eczema in children. Br J Dermatol, 125(6): 604.

Green, C., J. L. Colquitt, et al. (2005). Topical corticosteroids for atopic eczema: clinical and cost effectiveness of once-daily vs. more frequent use. Br J Dermatol, 152(1): 130-141.

Haigh, J. M. and E. W. Smith (1991). Topical corticosteroid-induced skin blanching measurement: eye or instrument? Arch Dermatol, 127(7): 1065. 
Hartmann, F. and J. J. Lahmann (1977). Effect of the external use of triamcinolone acetonide under foil occlussive dressings on the plasma cortisol level and on the diurnal rhythm of plasma cortisol. Hautarzt, 28(4): 198-202.

Hindley, D., G. Galloway, et al. (2006). A randomised study of "wet wraps" versus conventional treatment for atopic eczema. Arch Dis Child, 91(2): 164-168.

Hon, K. L., K. Y. Wong, et al. (2007). Efficacy and problems associated with using a wetwrap garment for children with severe atopic dermatitis. J Dermatolog Treat, 18(5): 301-305.

Kizu, J., W. Ichihara, et al. (2004). Survey of mixing commercially available corticosteroid ointments with other ointments and the anti-inflammatory activity of the admixtures. Yakugaku Zasshi, 124(2): 93-97.

Krakowski, A. C., L. F. Eichenfield, et al. (2008). Management of atopic dermatitis in the pediatric population. Pediatrics, 122(4): 812-824.

Lagos, B. R. and H. I. Maibach (1998). Frequency of application of topical corticosteroids: an overview. Br J Dermatol, 139(5): 763-766.

Lee, J. H., S. J. Lee, et al. (2007). The effect of wet-wrap dressing on epidermal barrier in patients with atopic dermatitis. J Eur Acad Dermatol Venereol, 21(10): 1360-1368.

Levin, C. and H. I. Maibach (2002). Topical corticosteroid-induced adrenocortical insufficiency: clinical implications. Am J Clin Dermatol, 3(3): 141-147.

Mallon, E., S. Powell, et al. (1994). Wet wrap' dressings for the treatment of atopic ezcema in the community. J Dermatolog Treat, (5): 97-87.

McGowan, R., P. Tucker, et al. (2003). Short-term growth and bone turnover in children undergoing occlusive steroid ('Wet-Wrap') dressings for treatment of atopic eczema. J Dermatolog Treat, 14(3): 149-152.

Nicol, N. H. (1987). Atopic dermatitis: the (wet) wrap-up. Am J Nurs, 87(12): 1560-1563.

Ohtani, M., N. Yamada, et al. (2002). Effect of admixture of commercially available corticosteroid ointments and/or creams on vasoconstrictor activity. Yakugaku Zasshi, 122(1): 107-112.

Ong, P. Y., R. M. Ferdman, et al. (2008). Down-regulation of atopic dermatitis-associated serum chemokines by wet-wrap treatment: a pilot study. Ann Allergy Asthma Immunol, 100(3): 286-287.

Ong, P. Y., T. Ohtake, et al. (2002). Endogenous antimicrobial peptides and skin infections in atopic dermatitis. $N$ Engl J Med, 347(15): 1151-1160.

Oranje, A. P., A. C. Devillers, et al. (2006). Treatment of patients with atopic dermatitis using wet-wrap dressings with diluted steroids and/or emollients. An expert panel's opinion and review of the literature. J Eur Acad Dermatol Venereol, 20(10): 1277-1286.

Oranje, A. P., R. Verbeek, et al. (2009). Wet-wrap treatment using dilutions of tacrolimus ointment and fluticasone propionate cream in human APOC1 $(+/+)$ mice with atopic dermatitis. Br J Dermatol, 160(1): 54-61.

Page, B. (2005). The benefits of Tubifast Garments in the management of atopic eczema. $\mathrm{Br}$ J Nurs, 14(5): 289-290, 292.

Park, K., F. Kamangar, et al. (2011). Instant Barrier Repair: A Pilot Study Investigating Occlusion with a New Hydrogel Patch for the Treatment of Atopic Dermatitis. J Dermatolog Treat, Accepted for publication. 
Pei, A. Y., H. H. Chan, et al. (2001). The effectiveness of wet wrap dressings using $0.1 \%$ mometasone furoate and $0.005 \%$ fluticasone proprionate ointments in the treatment of moderate to severe atopic dermatitis in children. Pediatr Dermatol, 18(4): 343-348.

Rajka, G., R. Aly, et al. (1981). The effect of short-term occlusion on the cutaneous flora in atopic dermatitis and psoriasis. Acta Derm Venereol, 61(2): 150-153.

Schnopp, C., C. Holtmann, et al. (2002). Topical steroids under wet-wrap dressings in atopic dermatitis--a vehicle-controlled trial. Dermatology, 204(1): 56-59.

Tang, W. Y. (2000). Diluted steroid facial wet wraps for childhood atopic eczema. Dermatology, 200(4): 338-339.

Thaci, D., K. Steinmeyer, et al. (2003). Occlusive treatment of chronic hand dermatitis with pimecrolimus cream $1 \%$ results in low systemic exposure, is well tolerated, safe, and effective. An open study. Dermatology, 207(1): 37-42.

Volden, G. (1992). Successful treatment of therapy-resistant atopic dermatitis with clobetasol propionate and a hydrocolloid occlusive dressing. Acta Derm Venereol Suppl (Stockh), 176: 126-128.

Williams, H. (2006). Wet wrap bandages for 4 weeks did not differ from topical ointments but increased skin infections in paediatric atopic eczema. Evid Based Med, 11(4): 108.

Williams, R. E. (2000). The antibacterial-corticosteroid combination. What is its role in atopic dermatitis? Am J Clin Dermatol, 1(4): 211-215.

Wolkerstorfer, A., R. L. Visser, et al. (2000). Efficacy and safety of wet-wrap dressings in children with severe atopic dermatitis: influence of corticosteroid dilution. $\mathrm{Br} \mathrm{J}$ Dermatol, 143(5): 999-1004.

Zhai, H. and H. I. Maibach (2001). Effects of skin occlusion on percutaneous absorption: an overview. Skin Pharmacol Appl Skin Physiol, 14(1): 1-10. 


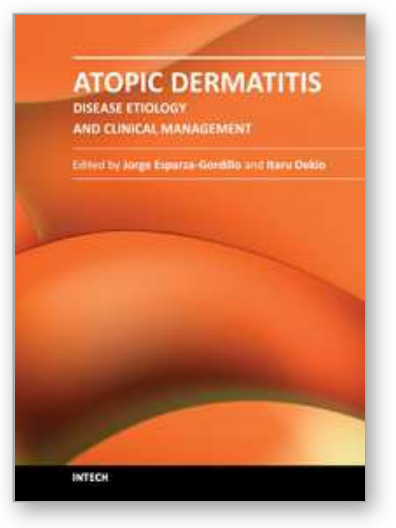

\author{
Atopic Dermatitis - Disease Etiology and Clinical Management \\ Edited by Dr. Jorge Esparza-Gordillo
}

ISBN 978-953-51-0110-9

Hard cover, 414 pages

Publisher InTech

Published online 22, February, 2012

Published in print edition February, 2012

Atopic Dermatitis is a common disease characterized by inflamed, itching and dry skin. This relapsing allergic disorder has complex etiology and shows a remarkably high clinical heterogeneity which complicates the diagnosis and clinical management. This book is divided into 4 sections. The first section (Disease Etiology) describes some of the physiological mechanisms underlying Atopic Dermatitis, including alterations in the immune system and the skin-barrier function. The important role of host-microorganism interactions on the pathophysiology of Atopic Dermatitis is discussed in the second section (Microorganisms in Atopic Dermatitis). An overview of the clinical diagnostic criteria and the disease management protocols commonly used is given in the third section (Diagnosis and Clinical Management). The last section (New Treatments) describes new therapeutic approaches that are not widely used but are currently being studied due to preliminary evidence showing a clinical benefit for Atopic Dermatitis.

\title{
How to reference
}

In order to correctly reference this scholarly work, feel free to copy and paste the following:

Misha M. Heller, Eric S. Lee, Faranak Kamangar, Wilson Liao and John Y. M. Koo (2012). Occlusive Therapy in Atopic Dermatitis, Atopic Dermatitis - Disease Etiology and Clinical Management, Dr. Jorge Esparza-Gordillo (Ed.), ISBN: 978-953-51-0110-9, InTech, Available from: http://www.intechopen.com/books/atopic-dermatitisdisease-etiology-and-clinical-management/occlusive-therapy-in-atopic-dermatitis

\section{INTECH}

open science | open minds

\section{InTech Europe}

University Campus STeP Ri

Slavka Krautzeka 83/A

51000 Rijeka, Croatia

Phone: +385 (51) 770447

Fax: +385 (51) 686166

www.intechopen.com

\section{InTech China}

Unit 405, Office Block, Hotel Equatorial Shanghai

No.65, Yan An Road (West), Shanghai, 200040, China

中国上海市延安西路65号上海国际贵都大饭店办公楼 405 单元

Phone: +86-21-62489820

Fax: $+86-21-62489821$ 
(C) 2012 The Author(s). Licensee IntechOpen. This is an open access article distributed under the terms of the Creative Commons Attribution 3.0 License, which permits unrestricted use, distribution, and reproduction in any medium, provided the original work is properly cited. 